\title{
Positive solutions of periodic boundary value problems for the second-order differential equation with a parameter
}

Ying Wang*, Jing Li and Zengxia Cai

\section{"Correspondence:} lywy1981@163.com School of Mathematics and Statistics, Linyi University, Linyi, Shandong 276000, People's Republic of China

\begin{abstract}
In this paper, we investigate the existence of positive solutions for a class of singular second-order differential equations with periodic boundary conditions. By using the fixed point theory in cones, the explicit range for $\lambda$ is derived such that for any $\lambda$ lying in this interval, the existence of at least one positive solution to the boundary value problem is guaranteed.
\end{abstract}

MSC: $34 \mathrm{~B} 16 ; 34 \mathrm{~B} 18$

Keywords: positive solution; periodic boundary conditions; second-order differential equation; singularity

\section{Introduction}

Reaction-diffusion problems often arise in physics, chemistry, biology, economics, and various engineering fields. A class of reaction-diffusion equations

$$
w_{t}=w_{x x}+H(w)
$$

includes several known evolution equations. For equation (1), if traveling wave satisfies $w(x, t)=W(x-C t)$ with speed $C$, then equation (1) can be converted to a second-order ordinary differential equation

$$
W^{\prime \prime}+C W^{\prime}+H(W)=0 \text {. }
$$

With appropriate boundary value conditions, the existence of positive solution of equation (2) is significant and helpful. The Liebau phenomenon, which is in honor of the physician Liebauh's pioneering work, is the occurrence of valveless pumping through the application of a periodic force at a place which lies asymmetric with respect to system configuration. Propst [1] made use of differential equations to model a periodically forced flow through different pipe-tank configurations. In one pipe-one tank configuration, ignore the singularity in the corresponding differential equation model, namely

$$
\left\{\begin{array}{l}
u^{\prime \prime}(t)+a u^{\prime}(t)+\frac{1}{u}\left(b\left(u^{\prime}(t)\right)^{2}-e(t)\right)+c=0, \quad t \in[0, T] \\
u(0)=u(T), \quad u^{\prime}(0)=u^{\prime}(T) .
\end{array}\right.
$$

(c) The Author(s) 2017. This article is distributed under the terms of the Creative Commons Attribution 4.0 International License (http://creativecommons.org/licenses/by/4.0/), which permits unrestricted use, distribution, and reproduction in any medium, provided you give appropriate credit to the original author(s) and the source, provide a link to the Creative Commons license, and indicate if changes were made. 
According to the physical meaning of the involved parameters, assume $a \geq 0, b>1, c>0$, and $e$ is continuous and $T$-periodic on $[0,+\infty)$. In [2], Cid et al. applied the substitution $u=x^{\mu}, \mu=\frac{1}{b+1}$, and then transformed the singular periodic boundary value problem (3) to the regular problem

$$
\left\{\begin{array}{l}
x^{\prime \prime}(t)+a x^{\prime}(t)+s(t) x^{\beta}-r(t) x^{\alpha}=0, \quad t \in[0, T] \\
x(0)=x(T), \quad x^{\prime}(0)=x^{\prime}(T),
\end{array}\right.
$$

where $r(t)=\frac{e(t)}{\mu}, s(t)=\frac{c}{\mu}, \alpha=1-2 \mu, \beta=1-\mu$. Based on the lower and upper solution technique, the existence and asymptotic stability of positive solutions for (3) are obtained.

In this paper, we discuss the positive solutions of the following periodic boundary value problem (PBVP):

$$
\left\{\begin{array}{l}
x^{\prime \prime}(t)+a x^{\prime}(t)+k^{2} x(t)=\lambda f(t, x(t)), \quad t \in(0, T), \\
x(0)=x(T), \quad x^{\prime}(0)=x^{\prime}(T),
\end{array}\right.
$$

where $a \geq 0, k \in(-\infty,+\infty), \lambda>0$ is a parameter, $f:(0, T) \times(0,+\infty) \rightarrow[0,+\infty)$ is a continuous function and $f(t, u)$ may be singular at $t=0, t=T$ and $u=0$.

In recent years, the existence of solutions for differential equations has been widely studied by many scholars in the mathematical sense (see [3-20] and the references therein). In $[3,4]$, through the use of Guo-Krasnosel'skii's fixed point theorem, the existence and multiplicity of positive solutions for the following periodic boundary value problem were established.

$$
\begin{aligned}
& \left\{\begin{array}{l}
-x^{\prime \prime}(t)+\rho^{2} x(t)=f(t, x(t)), \quad t \in[0,2 \pi], \rho>0, \\
x(0)=x(2 \pi), \quad x^{\prime}(0)=x^{\prime}(2 \pi),
\end{array}\right. \\
& \left\{\begin{array}{l}
x^{\prime \prime}(t)+\rho^{2} x(t)=f(t, x(t)), \quad t \in[0,2 \pi], 0<\rho<\frac{1}{2}, \\
x(0)=x(2 \pi), \quad x^{\prime}(0)=x^{\prime}(2 \pi) .
\end{array}\right.
\end{aligned}
$$

In [5], the author researched PBVP (8) by using an $L^{p}$-anti-maximum principle and obtained the existence results in order to overcome the difficulties of the symbol of Green's functions for the corresponding linear periodic problem:

$$
\left\{\begin{array}{l}
x^{\prime \prime}(t)+a(t) x(t)=f(x(t))+a(t) x(t), \quad t \in[0, T], \\
x(0)=x(2 T), \quad x^{\prime}(0)=x^{\prime}(2 T) .
\end{array}\right.
$$

Motivated by the above works, we consider PBVP (5). In (5), if $f(t, x(t))=m^{2} x(t)+s(t) x^{\beta}-$ $r(t) x^{\alpha}$, then (4) is a special case of (5). Compared with [3, 4], in which the existence and multiplicity of positive solutions for (6) (7) are considered, we not only obtain the existence of positive solutions for (5), but also increase the parameter $\lambda$ and get the explicit range of $\lambda$ by using the fixed point theory in cones. Therefore, our article contains, promotes, and improves the previous results to a certain extent. 


\section{Preliminaries and lemmas}

In this section, we present some notations and lemmas that will be used in the proof of our main results.

Lemma 2.1 ([21, 22]) Let $h \in C(0, T) \cap L^{1}(0, T)$, then the boundary value problem

$$
\left\{\begin{array}{l}
x^{\prime \prime}(t)+a x^{\prime}(t)+k^{2} x(t)=h(t), \quad t \in[0, T] \\
x(0)=x(T), \quad x^{\prime}(0)=x^{\prime}(T)
\end{array}\right.
$$

has an integral representation

$$
x(t)=\int_{0}^{T} G(t, s) h(s) d s
$$

where $G(t, s)$ is the related Green's function.

Lemma 2.2 ([23]) Assume that condition $\left(\mathbf{H}_{0}\right)$ holds,

$\left(\mathbf{H}_{0}\right) k>0, k^{2}<\left(\frac{\pi}{T}\right)^{2}+\left(\frac{a}{2}\right)^{2}$.

Then $G(t, s)$ has the following properties:

(i) $G(t, s)>0,(t, s) \in[0, T] \times[0, T]$;

(ii) $\int_{0}^{T} G(t, s) d s=\frac{1}{k^{2}}$;

(iii) There exists a constant $\xi \in(0,1)$ such that $G(t, s) \geq G(s, s) \geq \xi G(t, s)$, $(t, s) \in[0, T] \times[0, T]$.

Let $X=C[0, T]$, then $X$ is a Banach space with the norm $\|x\|=\max _{t \in[0, T]}|x(t)|$. Denote

$$
K=\{x \in X: x(t) \geq \xi\|x\|, t \in[0, T]\},
$$

where $\xi$ is defined as Lemma 2.2. It is easy to see that $K$ is a positive and normal cone in $X$. For any $0<r<R<+\infty$, let $K_{r, R}=\{x \in K: r \leq\|x\| \leq R\}$. In this paper, we always assume that the following conditions hold.

$\left(\mathbf{H}_{1}\right) f:(0, T) \times(0,+\infty) \rightarrow[0,+\infty)$ is a continuous function and

$$
f(t, u) \leq \phi(t)(g(u)+h(u)), \quad(t, u) \in(0, T) \times(0,+\infty),
$$

where $\phi:(0, T) \rightarrow[0,+\infty)$ is continuous and singular at $t=0, T, \phi(t) \not \equiv 0$ on $[0,+\infty)$, $g:(0,+\infty) \rightarrow[0,+\infty)$ is continuous and nonincreasing, $h:[0,+\infty) \rightarrow[0,+\infty)$ is continuous.

$\left(\mathbf{H}_{2}\right) \int_{0}^{T} G(s, s) \phi(s) d s<+\infty$.

Under assumptions $\left(\mathbf{H}_{0}\right)-\left(\mathbf{H}_{2}\right)$, for any $n \in \mathbb{N}, \mathbb{N}$ is a natural number set, we define a nonlinear integral operator $A_{n}: K \rightarrow X$ by

$$
\left(A_{n} x\right)(t)=\lambda \int_{0}^{T} G(t, s) f_{n}(s, x(s)) d s, \quad t \in[0, T]
$$


where $f_{n}(t, u)=f\left(t,\left(u+\frac{1}{n}\right)\right)$. Obviously, the existence of solutions to (5) is equivalent to the existence of solutions in $K$ for the operator equation $A_{n} x=x$ defined by (10). In this paper, the proof of the main theorem is based on the fixed point theory in cones. We list the following lemmas which are needed in our study.

Lemma 2.3 ([24]) Let $K$ be a positive cone in a real Banach space X. Denote $K_{r}=\{x \in K$ : $\|x\|<r\}, \bar{K}_{r, R}=\{x \in K: r \leq\|x\| \leq R\}, 0<r<R<+\infty$. Let $A: \bar{K}_{r, R} \rightarrow K$ be a completely continuous operator. If the following conditions are satisfied:

(1) $\|A x\| \leq\|x\|, \forall x \in \partial K_{R}$;

(2) There exists $x_{0} \in \partial K_{1}$ such that $x \neq A x+m x_{0}, \forall x \in \partial K_{r}, m>0$.

Then $A$ has fixed points in $\bar{K}_{r, R}$.

Remark 2.1 If (1) and (2) are satisfied for $x \in \partial K_{r}$ and $x \in \partial K_{R}$, respectively, then Lemma 2.3 is still true.

Lemma 2.4 ([25]) Let $K$ be a positive cone in a Banach space $E, \Omega_{1}$ and $\Omega_{2}$ be bounded open sets in $E, \theta \in \Omega_{1}, \bar{\Omega}_{1} \subset \Omega_{2}, A: K \cap \bar{\Omega}_{2} \backslash \Omega_{1} \rightarrow$ P be a completely continuous operator. If the following conditions are satisfied:

$$
\|A x\| \leq\|x\|, \quad \forall x \in K \cap \partial \Omega_{1}, \quad\|A x\| \geq\|x\|, \quad \forall x \in K \cap \partial \Omega_{2},
$$

or

$$
\|A x\| \geq\|x\|, \quad \forall x \in K \cap \partial \Omega_{1}, \quad\|A x\| \leq\|x\|, \quad \forall x \in K \cap \partial \Omega_{2},
$$

then $A$ has at least one fixed point in $K \cap\left(\bar{\Omega}_{2} \backslash \Omega_{1}\right)$.

\section{Main results}

Theorem 3.1 Assume that $\left(\mathbf{H}_{0}\right)-\left(\mathbf{H}_{2}\right)$ hold, then $A_{n}: K \rightarrow K$ is a completely continuous operator for any fixed $n \in \mathbb{N}$.

Proof Let $\lambda>0$ and $n \in \mathbb{N}$ be fixed. For any $x \in K$ and $t \in[0, T]$, by Lemma 2.2, we have

$$
\begin{aligned}
\lambda \int_{0}^{T} G(s, s) f_{n}(s, x(s)) d s & \leq\left(A_{n} x\right)(t)=\lambda \int_{0}^{T} G(t, s) f_{n}(s, x(s)) d s \\
& \leq \frac{\lambda}{\xi} \int_{0}^{T} G(t, s) f_{n}(s, x(s)) d s .
\end{aligned}
$$

This implies that $\left(A_{n} x\right)(t) \geq \xi\left\|A_{n} x\right\|$, therefore $A_{n}(K) \subset K$. By a standard argument, under assumptions $\left(\mathbf{H}_{0}\right)-\left(\mathbf{H}_{2}\right)$, we know that $A_{n}: K \rightarrow K$ is well defined.

Next, for any positive integers $n, m \in \mathbb{N}$, we define an operator $A_{n, m}: K \rightarrow X$ by

$$
\left(A_{n, m} x\right)(t)=\lambda \int_{\frac{1}{m}}^{T-\frac{1}{m}} G(t, s) f_{n}(s, x(s)) d s, \quad t \in[0, T] .
$$

In a similar discussion, $A_{n, m}: K \rightarrow X$ is well defined and $A_{n, m}(K) \subseteq K$. In what follows, we will prove that $A_{n, m}: K \rightarrow K$ is completely continuous for each $m \geq 1$. Firstly, we show that 
$A_{n, m}: K \rightarrow K$ is continuous. Let $x_{v}, x \in K$ satisfy $\left\|x_{v}-x\right\| \rightarrow 0$ as $v \rightarrow+\infty$. Notice that $t \in\left[\frac{1}{m}, T-\frac{1}{m}\right],\left|f_{n}\left(t, x_{v}(t)\right)-f_{n}(t, x(t))\right| \rightarrow 0$ as $v \rightarrow+\infty$. Using the Lebesgue dominated convergence theorem, we have

$$
\begin{aligned}
& \left|\lambda \int_{\frac{1}{m}}^{T-\frac{1}{m}} G(t, s) f_{n}\left(s, x_{v}(s)\right) d s-\lambda \int_{\frac{1}{m}}^{T-\frac{1}{m}} G(t, s) f_{n}(s, x(s)) d s\right| \\
& \quad \leq \frac{\lambda}{\xi} \int_{\frac{1}{m}}^{T-\frac{1}{m}} G(s, s)\left|f_{n}\left(s, x_{v}(s)\right)-f_{n}(s, x(s))\right| d s \rightarrow 0, \quad v \rightarrow+\infty .
\end{aligned}
$$

Therefore

$$
\left\|A_{n, m} x_{v}-A_{n, m} x\right\| \leq \frac{\lambda}{\xi} \int_{\frac{1}{m}}^{T-\frac{1}{m}} G(s, s)\left|f_{n}\left(s, x_{v}(s)\right)-f_{n}(s, x(s))\right| d s \rightarrow 0, \quad v \rightarrow+\infty .
$$

So, $A_{n, m}: K \rightarrow C[0, T]$ is continuous for any natural numbers $n, m$. Then $A_{n, m}: K \rightarrow K$ is continuous for any natural numbers $n, m$.

Let $D \subset K$ be any bounded set, then for any $x \in D$, we have $\|x\| \leq r$, and then $0<\xi r \leq$ $x(t) \leq r$ for any $t \in[0, T]$. By $\left(\mathbf{H}_{1}\right)-\left(\mathbf{H}_{2}\right)$, for any $x \in D$, we have

$$
\begin{aligned}
& \left|\lambda \int_{\frac{1}{m}}^{T-\frac{1}{m}} G(t, s) f_{n}(s, x(s)) d s\right| \\
& \quad \leq \frac{\lambda}{\xi} \int_{\frac{1}{m}}^{T-\frac{1}{m}} G(s, s) \phi(s)\left(g\left(x(s)+\frac{1}{n}\right)+h\left(x(s)+\frac{1}{n}\right)\right) d s \\
& \quad \leq \frac{\lambda}{\xi} \int_{\frac{1}{m}}^{T-\frac{1}{m}} G(s, s) \phi(s)\left(g\left(\xi r+\frac{1}{n}\right)+h\left(x(s)+\frac{1}{n}\right)\right) d s \\
& \quad \leq \frac{\lambda}{\xi} \int_{\frac{1}{m}}^{T-\frac{1}{m}} G(s, s) \phi(s)\left(g(\xi r)+\max _{y \in[\xi r, r+1]} h(y)\right) d s \\
& \quad<+\infty .
\end{aligned}
$$

So, $A_{n, m} D$ is bounded in $K$.

In order to show that $A_{n, m}$ is a compact operator, we only need to show that $A_{n, m} D$ is equicontinuous. For any $\varepsilon>0$, by the continuity of $G(t, s)$ on $[0, T] \times[0, T]$, there exists $\delta>0$ such that for any $t_{1}, t_{2} \in[0, T], s \in\left[\frac{1}{m}, T-\frac{1}{m}\right]$, and $\left|t_{1}-t_{2}\right|<\delta$, we have

$$
\left|G\left(t_{1}, s\right)-G\left(t_{2}, s\right)\right|<\varepsilon\left(\lambda \int_{\frac{1}{m}}^{T-\frac{1}{m}} \phi(s)\left(g(\xi r)+\max _{y \in[\xi r, r+1]} h(y)\right) d s\right)^{-1} .
$$

Then, for any $x \in D$, for any $t_{1}, t_{2} \in[0, T], s \in\left[\frac{1}{m}, T-\frac{1}{m}\right]$, and $\left|t_{1}-t_{2}\right|<\delta$, we have

$$
\begin{aligned}
& \left|\left(A_{n, m}\right)\left(t_{1}\right)-\left(A_{n, m}\right)\left(t_{2}\right)\right| \\
& \quad \leq \lambda \int_{\frac{1}{m}}^{T-\frac{1}{m}}\left|G\left(t_{1}, s\right)-G\left(t_{2}, s\right)\right| f_{n}(s, x(s)) d s \\
& \quad \leq \lambda \int_{\frac{1}{m}}^{T-\frac{1}{m}}\left|G\left(t_{1}, s\right)-G\left(t_{2}, s\right)\right| \phi(s)\left(g\left(x(s)+\frac{1}{n}\right)+h\left(x(s)+\frac{1}{n}\right)\right) d s
\end{aligned}
$$




$$
\begin{aligned}
& \leq \lambda \int_{\frac{1}{m}}^{T-\frac{1}{m}}\left|G\left(t_{1}, s\right)-G\left(t_{2}, s\right)\right| \phi(s)\left(g(\xi r)+\max _{y \in[\xi r, r+1]} h(y)\right) d s \\
& <\varepsilon
\end{aligned}
$$

which means that $A_{n, m} D$ is equicontinuous. By the Arzela-Ascoli theorem, $A_{n, m} D$ is a relatively compact set and so $A_{n, m}: K \rightarrow K$ is a completely continuous operator.

Finally, we show that $A_{n}: K \rightarrow K$ is a completely continuous operator. For any $t \in[0, T]$ and $x \in S=\{x \in K,\|x\| \leq 1\}$, by (10), (11), we have

$$
\begin{aligned}
\lambda & \int_{0}^{\frac{1}{m}} G(t, s) f_{n}(s, x(s)) d s+\lambda \int_{T-\frac{1}{m}}^{T} G(t, s) f_{n}(s, x(s)) d s \\
& \leq \frac{\lambda}{\xi}\left(\int_{0}^{\frac{1}{m}}+\int_{T-\frac{1}{m}}^{T}\right) G(s, s) \phi(s)\left(g\left(x(s)+\frac{1}{n}\right)+h\left(x(s)+\frac{1}{n}\right)\right) d s \\
& \leq \frac{\lambda}{\xi}\left(\int_{0}^{\frac{1}{m}}+\int_{T-\frac{1}{m}}^{T}\right) G(s, s) \phi(s)\left(g\left(\frac{1}{n}\right)+\max _{y \in\left[\frac{1}{n}, 2\right]} h(y)\right) d s \\
& \rightarrow 0, \quad m \rightarrow+\infty .
\end{aligned}
$$

Hence

$$
\left\|A_{n}-A_{n, m}\right\|=\sup _{x \in S}\left\|A_{n} x-A_{n, m} x\right\| \rightarrow 0, \quad m \rightarrow+\infty .
$$

Therefore, by $A_{n, m}: K \rightarrow K$ is a completely continuous operator, we get that $A_{n}: K \rightarrow K$ is a completely continuous operator.

Theorem 3.2 Assume that $\left(\mathbf{H}_{0}\right)-\left(\mathbf{H}_{2}\right)$ hold and $f$ satisfies the following condition:

$\left(\mathbf{H}_{3}\right)$ There exists $[a, b] \subset(0, T)$ such that

$$
\lim _{u \rightarrow+\infty} \min _{t \in[a, b]} \frac{f(t, u)}{u}=+\infty
$$

Then there exists $\bar{\lambda}>0$ such that PBVP (5) has at least one positive solution for any $\lambda \in$ $(0, \bar{\lambda})$.

Proof Choose $r_{1}>0$, let

$$
\bar{\lambda}=\min \left\{1, \frac{\xi r_{1}}{\int_{0}^{T} G(s, s) \phi(s)\left(g(\xi r)+\max _{y \in\left[\xi r_{1}, r_{1}+1\right]} h(y)\right) d s}\right\}
$$

Let $K_{r_{1}}=\left\{x \in K:\|x\|<r_{1}\right\}$. For any $x \in \partial K_{r_{1}}, t \in[0, T]$, by the definition of $\|\cdot\|$, we have

$$
x(t) \leq\|x\| \leq r_{1}, \quad x(t) \geq \xi\|x\| \geq \xi r_{1} .
$$

For any $\lambda \in(0, \bar{\lambda})$, we have

$$
\begin{aligned}
\left|\left(A_{n} x\right)(t)\right| & =\left|\lambda \int_{0}^{T} G(t, s) f_{n}(s, x(s)) d s\right| \\
& \leq \frac{\lambda}{\xi} \int_{0}^{T} G(s, s) \phi(s)\left(g\left(x(s)+\frac{1}{n}\right)+h\left(x(s)+\frac{1}{n}\right)\right) d s
\end{aligned}
$$




$$
\begin{aligned}
& \leq \frac{\lambda}{\xi} \int_{0}^{T} G(s, s) \phi(s)\left(g\left(\xi r+\frac{1}{n}\right)+h\left(x(s)+\frac{1}{n}\right)\right) d s \\
& \leq \frac{\lambda}{\xi} \int_{0}^{T} G(s, s) \phi(s)\left(g(\xi r)+\max _{y \in\left[\xi r_{1}, r_{1}+1\right]} h(y)\right) d s \\
& <r_{1} .
\end{aligned}
$$

Thus,

$$
\left\|A_{n} x\right\| \leq\|x\| \quad \text { for any } x \in \partial K_{r_{1}} .
$$

On the other hand, by the inequality in $\left(\mathbf{H}_{3}\right)$, choose $l>0$ such that $\lambda l \xi r_{2} \int_{a}^{b} G(s, s) d s>1$, then there exists $N^{*}>0$ such that

$$
f(t, u) \geq l u, \quad u \geq N^{*}, \quad t \in[a, b] .
$$

Let $r_{2}>\max \left\{r_{1}, \frac{N^{*}}{\xi}\right\}, K_{r_{2}}=\left\{x \in K:\|x\|<r_{2}\right\}$. Take $q_{1} \equiv 1 \in \partial K_{1}=\{x \in K:\|x\|=1\}$. For any $x \in \partial K_{r_{2}}, \mu>0, n \in \mathbb{N}$, we will show

$$
x \neq A_{n} x+\mu q_{1}
$$

Otherwise, there exist $x_{0} \in \partial K_{r_{2}}$ and $\mu_{0}>0$ such that $x_{0}=A_{n} x_{0}+\mu_{0} q_{1}$. From $x_{0} \in \partial K_{r_{2}}$, we know that $\left\|x_{0}\right\|=r_{2}$. Then, for $t \in[a, b]$, we have

$$
x(t) \geq \xi\|x\| \geq \xi r_{2} \geq N^{*} .
$$

Hence, we conclude that

$$
\begin{aligned}
x_{0}(t) & =\lambda \int_{0}^{T} G(t, s) f_{n}\left(s, x_{0}(s)\right) d s+\mu_{0} \\
& \geq \lambda \int_{a}^{b} G(s, s) f_{n}\left(s, x_{0}(s)\right) d s+\mu_{0} \\
& \geq \lambda \int_{a}^{b} G(s, s) l \xi r_{2} d s+\mu_{0} \\
& \geq r_{2}+\mu_{0}>r_{2} .
\end{aligned}
$$

This implies that $r_{2}>r_{2}$, which is a contradiction. This yields that (14) holds.

It follows from the above discussion, (13), (14), Lemma 2.3 and Theorem 3.1 that, for any $n \in \mathbb{N}, \lambda \in(0, \bar{\lambda}), A_{n}$ has a fixed point $x_{n} \in \bar{K}_{r_{2}} \backslash K_{r_{1}}$.

Let $\left\{x_{n}\right\}_{n=1}^{\infty}$ be the sequence of solutions of PBVP (5). It is easy to see that they are uniformly bounded. From $x_{n} \in \bar{K}_{r_{2}} \backslash K_{r_{1}}$, we know that

$$
r_{2} \geq\left\|x_{n}\right\| \geq x_{n}(t) \geq \xi\left\|x_{n}\right\| \geq \xi r_{1}, \quad t \in[0, T]
$$

For any $\varepsilon>0$, by the continuity of $G(t, s)$ on $[0, T] \times[0, T]$, there exists $\delta_{1}>0$ such that for any $t_{1}, t_{2}, s \in[0, T],\left|t_{1}-t_{2}\right|<\delta_{1}$, we have

$$
\left|G\left(t_{1}, s\right)-G\left(t_{2}, s\right)\right|<\varepsilon\left(\lambda \int_{0}^{T} \phi(s)\left(g\left(\xi r_{1}\right)+\max _{y \in\left[\xi r_{1}, r_{2}+1\right]} h(y)\right) d s\right)^{-1} .
$$


Then, for any $t_{1}, t_{2}, s \in[0, T],\left|t_{1}-t_{2}\right|<\delta_{1}$, we obtain

$$
\begin{aligned}
& \left|x_{n}\left(t_{1}\right)-x_{n}\left(t_{2}\right)\right| \\
& \quad \leq \lambda \int_{0}^{T}\left|G\left(t_{1}, s\right)-G\left(t_{2}, s\right)\right| f_{n}\left(s, x_{n}(s)\right) d s \\
& \quad \leq \lambda \int_{0}^{T}\left|G\left(t_{1}, s\right)-G\left(t_{2}, s\right)\right| \phi(s)\left(g\left(x_{n}(s)+\frac{1}{n}\right)+h\left(x_{n}(s)+\frac{1}{n}\right)\right) d s \\
& \quad \leq \lambda \int_{0}^{T}\left|G\left(t_{1}, s\right)-G\left(t_{2}, s\right)\right| \phi(s)\left(g\left(\xi r_{1}\right)+\max _{y \in\left[\xi r_{1}, r_{2}+1\right]} h(y)\right) d s \\
& \quad<\varepsilon .
\end{aligned}
$$

Similarly to (12), together with (15), by the Ascoli-Arzela theorem, the sequence $\left\{x_{n}\right\}_{n=1}^{\infty}$ has a subsequence being uniformly convergent on $[0, T]$. Without loss of generality, we still assume that $\left\{x_{n}\right\}_{n=1}^{\infty}$ itself uniformly converges to $x$ on $[0, T]$. Since $\left\{x_{n}\right\}_{n=1}^{\infty} \in \bar{K}_{r_{2}} \backslash K_{r_{1}} \subset K$, we have $x_{n} \geq 0$. Besides, we have

$$
\begin{aligned}
x_{n}(t)= & x_{n}\left(\frac{1}{2}\right)+x_{n}^{\prime}\left(\frac{1}{2}\right)\left(t-\frac{1}{2}\right)-a \int_{\frac{1}{2}}^{t}\left(x_{n}(s)-x_{n}\left(\frac{1}{2}\right)\right) d s \\
& -k^{2} \int_{\frac{1}{2}}^{t} \int_{\frac{1}{2}}^{s}\left(x_{n}(\varsigma)-\lambda f_{n}\left(\varsigma, x_{n}(\varsigma)\right) d \varsigma d s, \quad t \in(0, T) .\right.
\end{aligned}
$$

Since $\left\{x_{n}^{\prime}\left(\frac{1}{2}\right)\right\}_{n=1}^{\infty}$ is bounded, without loss of generality, we may assume $x_{n}^{\prime}\left(\frac{1}{2}\right) \rightarrow c_{0}$ as $n \rightarrow$ $+\infty$. Then, by (16) and the Lebesgue dominated convergence theorem, we have

$$
\begin{aligned}
x(t)= & x\left(\frac{1}{2}\right)+c_{0}\left(t-\frac{1}{2}\right)-a \int_{\frac{1}{2}}^{t}\left(x(s)-x\left(\frac{1}{2}\right)\right) d s \\
& -k^{2} \int_{\frac{1}{2}}^{t} \int_{\frac{1}{2}}^{s}(x(\varsigma)-\lambda f(\varsigma, x(\varsigma)) d \varsigma d s, \quad t \in(0, T) .
\end{aligned}
$$

By (17), the direct computation shows that

$$
x^{\prime \prime}(t)+a x^{\prime}(t)+k^{2} x(t)=\lambda f(t, x(t)), \quad t \in(0, T) .
$$

On the other hand, let $n \rightarrow+\infty$ in the following boundary conditions:

$$
x_{n}(0)=x_{n}(T), \quad x_{n}^{\prime}(0)=x_{n}^{\prime}(T)
$$

Therefore, we deduce that $x$ is a solution of PBVP (5). The proof is completed.

Theorem 3.3 Assume that $\left(\mathbf{H}_{0}\right)-\left(\mathbf{H}_{2}\right)$ hold and $f$ satisfies the following condition:

$\left(\mathbf{H}_{4}\right)$ There exists $[c, d] \subset(0, T)$ such that

$$
\liminf _{u \rightarrow+\infty} \min _{t \in[c, d]} f(t, u)>\frac{1}{\int_{c}^{d} G(s, s) d s}, \quad \lim _{u \rightarrow+\infty} \frac{h(u)}{u}=0
$$


Then there exists $\bar{\lambda}>0$ such that PBVP (5) has at least one positive solution for any $\lambda \in$ $(\bar{\lambda},+\infty)$.

Proof By the first inequality of $\left(\mathbf{H}_{4}\right)$, we have that there exists $N_{*}>0$ such that for any $t \in[c, d], u>N_{*}$, we have

$$
f(t, u)>\frac{1}{\int_{c}^{d} G(s, s) d s} .
$$

Select $\bar{\lambda}=\max \left\{1, \frac{N_{*}}{\xi}\right\}$. In the following proof, we suppose $\lambda>\bar{\lambda}$, choose $R_{1}=\lambda, K_{R_{1}}=\{x \in$ $\left.K:\|x\|<R_{1}\right\}$. For any $x \in \partial K_{R_{1}}, t \in[c, d]$, we have

$$
x(t) \geq \xi\|x\| \geq \xi R_{1}>N_{*} .
$$

Then, by (18), we have

$$
\begin{aligned}
\left|\left(T x_{n}\right)(t)\right| & =\lambda \int_{0}^{T} G(t, s) f_{n}(s, x(s)) d s \geq \lambda \int_{c}^{d} G(s, s) f_{n}(s, x(s)) d s \\
& \geq \lambda \int_{c}^{d} G(s, s) \frac{1}{\int_{c}^{d} G(s, s)} d s \geq R_{1} .
\end{aligned}
$$

Therefore, we have

$$
\left\|A_{n} x\right\| \geq\|x\| \quad \text { for any } x \in \partial K_{R_{1}} .
$$

Based on the second inequality in $\left(\mathbf{H}_{4}\right)$ and the continuity of $h(u)$ on $[0,+\infty)$, for

$$
\bar{c}=\max \left\{1,\left(\frac{4 \lambda}{\xi} \int_{0}^{T} G(s, s) \phi(s) d s\right)^{-1}\right\},
$$

there exists $N^{*}>0$ such that when $x \geq N^{*}$, for any $0 \leq z \leq x$, we have $h(z) \leq \bar{c} x$. Select

$$
R_{2} \geq\left\{2, R_{1}, N^{*}, \frac{2 \lambda}{\xi} \int_{0}^{T} G(s, s) \phi(s) g\left(\xi R_{2}\right) d s\right\} .
$$

Then, for any $x \in \partial K_{R_{2}}, t \in[0,+\infty)$, we have

$$
x(t) \leq\|x\| \leq R_{2}, \quad x(t) \geq \xi\|x\| \geq \xi R_{2} .
$$

Hence, we gain

$$
\begin{aligned}
\left|\left(A_{n} x\right)(t)\right| & =\left|\lambda \int_{0}^{T} G(t, s) f_{n}(s, x(s)) d s\right| \\
& \leq \frac{\lambda}{\xi} \int_{0}^{T} G(s, s) \phi(s)\left(g\left(x(s)+\frac{1}{n}\right)+h\left(x(s)+\frac{1}{n}\right)\right) d s \\
& \leq \frac{\lambda}{\xi} \int_{0}^{T} G(s, s) \phi(s)\left(g\left(\xi R_{2}+\frac{1}{n}\right)+h\left(x(s)+\frac{1}{n}\right)\right) d s \\
& \leq \frac{\lambda}{\xi} \int_{0}^{T} G(s, s) \phi(s)\left(g\left(\xi R_{2}\right)+\bar{c}\left(R_{2}+1\right)\right) d s \leq R_{2} .
\end{aligned}
$$


Thus,

$$
\left\|A_{n} x\right\| \leq\|x\| \quad \text { for any } x \in \partial K_{R_{2}} .
$$

It follows from the above discussion, (19), (20), Lemma 2.4 and Theorem 3.1 that, for $n \in \mathbb{N}, \lambda \in(\bar{\lambda},+\infty), A_{n}$ has a fixed point $x_{n} \in \bar{K}_{R_{2}} \backslash K_{R_{1}}$ satisfying $R_{1} \leq\left\|x_{n}\right\| \leq R_{2}$. The rest of the proof is similar to Theorem 3.2. That is the proof of Theorem 3.3.

Corollary 3.1 The conclusion of Theorem 3.3 is valid if $\left(\mathbf{H}_{4}\right)$ is replaced by the following:

$\left(\mathbf{H}_{4}^{*}\right)$ There exists $[c, d] \subset(0, T)$ such that

$$
\liminf _{u \rightarrow+\infty} \min _{t \in[c, d]} f(t, u)=+\infty, \quad \lim _{u \rightarrow+\infty} \frac{h(u)}{u}=0 .
$$

Remark 3.1 From the proof of Theorems 3.2 and 3.3, we can obtain the main results under the condition that the function $f(t, u)$ not only has singularity on $t$ but also has singularity on $u$, and we use the approximation method to overcome the difficulty caused by singularity.

Remark 3.2 In this paper, we can get the positive solution of PBVP (5) when the parameter $\lambda$ is sufficiently large and small; concretely, we can choose $\lambda \in(0,1)$ and $\lambda \in(1,+\infty)$. What is more, the solution $x$ in PBVP (5) satisfies $x(t)>0$ for any $t \in[0, T]$.

\section{Examples}

Consider the PBVP

$$
\left\{\begin{array}{l}
x^{\prime \prime}(t)+\frac{1}{2} x^{\prime}(t)+x(t)=\lambda f(t, x(t)), \quad t \in[0,1], \\
x(0)=x(1), \quad x^{\prime}(0)=x^{\prime}(1),
\end{array}\right.
$$

where $a=\frac{1}{2}, k=1, T=1$. Obviously, $\left(\mathbf{H}_{0}\right)$ holds. Take $f(t, u)=\frac{1}{\sqrt{t(1-t)}}\left(\frac{1}{\sqrt{u}}+u^{2}\right)$, we can suppose $\phi(t)=\frac{1}{\sqrt{t(1-t)}}, g(u)=\frac{1}{\sqrt{u}}, h(u)=u^{2}$. Since the continuous function $G(t, s)$ is positive for all $t, s \in[0, T]$, there exist constants $C_{1}>0, C_{2}>0$ such that $0<C_{1}<G(t, s)<C_{2}$ for all $t, s \in[0, T]$. Together with $G(t, s) \geq G(s, s) \geq \xi G(t, s),(t, s) \in[0, T] \times[0, T]$ in Lemma 2.2, we have $0<\xi C_{1}<G(s, s)<C_{2}$, so we can get

$$
\begin{aligned}
\int_{0}^{T} G(s, s) \phi(s) d s & =\int_{0}^{1} G(s, s) \phi(s) d s<C_{2} \int_{0}^{1} \frac{1}{\sqrt{s(1-s)}} d s \\
& =C_{2} \int_{0}^{1} \frac{1}{\sqrt{\frac{1}{4}-\left(s-\frac{1}{2}\right)^{2}}} d s=C_{2} \int_{0}^{1} \frac{2}{\sqrt{1-\left(2\left(s-\frac{1}{2}\right)\right)^{2}}} d s \\
& =C_{2} \int_{-1}^{1} \frac{1}{\sqrt{1-u^{2}}} d u\left(u=2\left(s-\frac{1}{2}\right)\right) \\
& =C_{2} \lim _{u \rightarrow 1} 2 \arcsin u=\pi<+\infty, \\
\lim _{u \rightarrow+\infty} \min _{t \in[a, b]} \frac{f(t, u)}{u} & =+\infty .
\end{aligned}
$$


So all the conditions of Theorem 3.2 are satisfied. By Theorem 3.2, PBVP (21) has at least one positive solution provided $\lambda$ is small enough.

\author{
Competing interests \\ The authors declare that they have no competing interests.
}

Authors' contributions

All authors typed, read, and approved the final manuscript.

\title{
Acknowledgements
}

The authors were supported financially by the National Natural Science Foundation of China (11626125), Natural Science Foundation of Shandong Province of China (ZR2016AP04), the Project of Shandong Province Higher Educational Science and Technology Program (J16LI03), the Science Research Foundation for Doctoral Authorities of Linyi University (LYDX2016BS080), and the Applied Mathematics Enhancement Program of Linyi University.

\section{Publisher's Note}

Springer Nature remains neutral with regard to jurisdictional claims in published maps and institutional affiliations.

Received: 8 October 2016 Accepted: 21 March 2017 Published online: 08 April 2017

\section{References}

1. Propst, G: Pumping effects in models of periodically forced flow configurations. Physica D 217, $193-201$ (2006)

2. Cid, JA, Propst, G, Tvrdy, M: On the pumping effect in a pipe/tank flow configuration with friction. Physica D 273(8), 28-33 (2014)

3. Jiang, D: On the existence of positive solutions to second order periodic BVPs. Acta Math. Sci. 18, 31-35 (1998)

4. Zhang, Z, Wang, J: On existence and multiplicity of positive solutions to periodic boundary value problems for singular nonlinear second order differential equations. J. Math. Anal. Appl. 281, 99-107 (2003)

5. Torres, PJ: Existence of one-signed periodic solutions of some second-order differential equations via a Krasnosel'skii fixed point theorem. J. Differ. Equ. 190, 643-662 (2003)

6. Zhang, Z, Wang, J: On existence and multiplicity of positive solutions to periodic boundary value problems for singular nonlinear second order differential equations. J. Math. Anal. Appl. 281, 99-107 (2003)

7. Yao, Q: Positive solutions of nonlinear second-order periodic boundary value problems. Appl. Math. Lett. 20, 583-590 (2007)

8. Fu, X, Wang, W: Periodic boundary value problems for second-order functional differential equations. J. Inequal. Appl. 2010, $11(2010)$

9. Wang, W, Shen, J, Nieto, JJ: Periodic boundary value problems for second order functional differential equations. J. Appl. Math. Comput. 36, 173-186 (2011)

10. Liu, L, Hao, X, Wu, Y: Positive solutions for singular second order differential equations with integral boundary conditions. Math. Comput. Model. 57, 836-847 (2013)

11. Liu, L, Sun, F, Zhang, X, Wu, Y: Bifurcation analysis for a singular differential system with two parameters via to degree theory. Nonlinear Anal., Model. Control 22(1), 31-50 (2017)

12. Zhang, $X, L i u, L, W u, Y$ : Fixed point theorems for the sum of three classes of mixed monotone operators and applications. Fixed Point Theory Appl. 2016, 49 (2016)

13. Liu, L, Zhang, $X$, Jiang, J, Wu, Y: The unique solution of a class of sum mixed monotone operator equations and its application to fractional boundary value problems. J. Nonlinear Sci. Appl. 9, 2943-2958 (2016)

14. Wu, J, Zhang, X, Liu, L, Wu, Y: Twin iterative solutions for a fractional differential turbulent flow model. Bound. Value Probl. 2016, $98(2016)$

15. Liu, L, Zhang, X, Liu, L, Wu, Y: Iterative positive solutions for singular nonlinear fractional differential equation with integral boundary conditions. Adv. Differ. Equ. 2016, 154 (2016)

16. Guo, L, Liu, L, Wu, Y: Existence of positive solutions for singular higher-order fractional differential equations with infinite-points boundary conditions. Bound. Value Probl. 2016, 114 (2016)

17. Guo, L, Liu, L, Wu, Y: Existence of positive solutions for singular fractional differential equations with infinite-point boundary conditions. Nonlinear Anal., Model. Control 21(5), 635-650 (2016)

18. Guo, L, Liu, L, Wu, Y: Uniqueness of iterative positive solutions for the singular fractional differential equations with integral boundary conditions. Bound. Value Probl. 2016, 147 (2016)

19. Jiang, J, Liu, L: Existence of solutions for a sequential fractional differential system with coupled boundary conditions. Bound. Value Probl. 2016, 147 (2016)

20. Bravyi, E: On solvability of periodic boundary value problems for second order linear functional differential equations. Electron. J. Qual. Theory Differ. Equ. (2016). doi:10.14232/ejqtde.2016.1.5

21. Cabada, A: Green's Functions in the Theory of Ordinary Differential Equations. Springer, Berlin (2014)

22. Cabada, A, Cid, JA, Máquez-Villamarín, B: Computation of Green's functions for boundary value problems with mathematica. Appl. Math. Comput. 219, 1919-1936 (2012)

23. Cid, JA, Infanteb, G, Tvrdýc, M, Zima, M: A topological approach to periodic oscillations related to the Liebau phenomenon. J. Math. Anal. Appl. 423, 1546-1556 (2015)

24. Amann, H: Fixed point equations and nonlinear eigenvalue problems in order Banach spaces. SIAM Rev. 18, 620-709 (1976)

25. Guo, DJ, Lakshmikantham, V: Nonlinear Problems in Abstract Cones. Academic Press, New York (1988) 